\title{
MERIDIAN SURFACES WITH CONSTANT MEAN CURVATURE IN PSEUDO-EUCLIDEAN 4-SPACE WITH NEUTRAL METRIC
}

\author{
BETÜL BULCA AND VELICHKA MILOUSHEVA
}

\begin{abstract}
In the present paper we consider a special class of Lorentz surfaces in the fourdimensional pseudo-Euclidean space with neutral metric which are one-parameter systems of meridians of rotational hypersurfaces with timelike or spacelike axis and call them meridian surfaces. We give the complete classification of minimal and quasi-minimal meridian surfaces. We also classify the meridian surfaces with non-zero constant mean curvature.
\end{abstract}

\section{INTRODUCTION}

The study of surfaces with constant mean curvature is one of the main topics in classical differential geometry which goes back to the latter part of the 18th century. Lagrange was the first who found the minimal surface equation in 1761 when he looked for a necessary condition for minimizing a certain integral. Actually, the notion of mean curvature was first formally defined by Meusnier in 1776. Throughout the 19th century grate mathematicians such as Gauss and Weierstrass devoted much of their studies to these surfaces. Constant mean curvature surfaces in the 3-dimensional Euclidean space are also studied intensively nowadays by many geometers for their physical interpretation. For example, surfaces with constant curvature are important mathematical models of soap films and soap bubbles.

Constant mean curvature surfaces (CMC surfaces) in arbitrary spacetime are important objects for the special role they play in the theory of general relativity. The study of CMC surfaces involves not only geometric methods but also PDE and complex analysis, that is why the theory of CMC surfaces is of great interest not only for mathematicians but also for physicists and engineers. Surfaces with constant mean curvature in Minkowski space have been studied intensively in the last years. See for example [16], [17, [20, [4], 3].

In the four-dimensional pseudo-Euclidean space with neutral metric very few results are known on surfaces with constant mean curvature. A special case of CMC surfaces are the quasi-minimal surfaces. A Lorentzian surface in a pseudo-Riemannian manifold is called quasi-minimal (pseudo-minimal or marginally trapped) if its mean curvature vector $H$ is lightlike at each point. Classification results on quasi-minimal surfaces in the pseudoEuclidean space $\mathbb{E}_{2}^{4}$ have been obtained recently. The classification of quasi-minimal surfaces with parallel mean curvature vector in $\mathbb{E}_{2}^{4}$ is given in [9]. In [5] B.-Y. Chen classified quasiminimal Lorentz flat surfaces in $\mathbb{E}_{2}^{4}$. As an application, he gave the complete classification of biharmonic Lorentz surfaces in $\mathbb{E}_{2}^{4}$ with lightlike mean curvature vector. Several other families of quasi-minimal surfaces have also been classified. For example, quasi-minimal surfaces with constant Gauss curvature in $\mathbb{E}_{2}^{4}$ were classified in [6, 11]. Quasi-minimal Lagrangian surfaces and quasi-minimal slant surfaces in complex space forms were classified, respectively, in [8] and [10]. For an up-to-date survey on quasi-minimal surfaces, see also [7].

In the present paper we construct special 2-dimensional Lorentz surfaces in $\mathbb{E}_{2}^{4}$ which are one-parameter systems of meridians of the rotational hypersurfaces with timelike or spacelike

2010 Mathematics Subject Classification. 53A35, 53B30, 53B25.

Key words and phrases. Meridian surfaces, quasi-minimal surfaces, constant mean curvature, pseudoEuclidean space with neutral metric. 
axis and call them meridian surfaces in $\mathbb{E}_{2}^{4}$. We describe all minimal meridian surfaces and show that all of them lie in hyperplanes of $\mathbb{E}_{2}^{4}$. We give the complete classification of quasiminimal meridian surfaces (Theorems 5, 6, and 7). We also classify the meridian surfaces with non-zero constant mean curvature (Theorems 8, 9, and 10).

\section{Preliminaries}

Let $\mathbb{E}_{2}^{4}$ be the 4-dimensional pseudo-Euclidean space with flat metric of index 2 given in local coordinates by

$$
\widetilde{g}=d x_{1}^{2}+d x_{2}^{2}-d x_{3}^{2}-d x_{4}^{2},
$$

where $\left(x_{1}, x_{2}, x_{3}, x_{4}\right)$ is a rectangular coordinate system of $\mathbb{E}_{2}^{4}$. We denote by $\langle.,$.$\rangle the indef-$ inite inner scalar product associated with $\widetilde{g}$. Since $\widetilde{g}$ is an indefinite metric, a vector $v \in \mathbb{E}_{2}^{4}$ can have one of the three casual characters: it can be spacelike if $\langle v, v\rangle>0$ or $v=0$, timelike if $\langle v, v\rangle\langle 0$, and lightlike if $\langle v, v\rangle=0$ and $v \neq 0$. This terminology is inspired by general relativity and the Minkowski 4-space $\mathbb{E}_{1}^{4}$.

We use the following denotations:

$$
\begin{aligned}
& \mathbb{S}_{2}^{3}(1)=\left\{V \in \mathbb{E}_{2}^{4}:\langle V, V\rangle=1\right\} ; \\
& \mathbb{H}_{1}^{3}(-1)=\left\{V \in \mathbb{E}_{2}^{4}:\langle V, V\rangle=-1\right\} .
\end{aligned}
$$

The space $\mathbb{S}_{2}^{3}(1)$ is known as the de Sitter space, and the space $\mathbb{H}_{1}^{3}(-1)$ is the hyperbolic space (or the anti-de Sitter space) [18.

Given a surface $M$ in $\mathbb{E}_{2}^{4}$, we denote by $g$ the induced metric of $\widetilde{g}$ on $M$. A surface $M$ in $\mathbb{E}_{2}^{4}$ is called Lorentz if the induced metric $g$ on $M$ is Lorentzian, i.e. at each point $p \in M$ we have the following decomposition

$$
\mathbb{E}_{2}^{4}=T_{p} M \oplus N_{p} M
$$

with the property that the restriction of the metric onto the tangent space $T_{p} M$ is of signature $(1,1)$, and the restriction of the metric onto the normal space $N_{p} M$ is of signature $(1,1)$.

We denote by $\nabla$ and $\bar{\nabla}$ the Levi-Civita connections of $M$ and $\mathbb{E}_{2}^{4}$, respectively. Then for any vector fields $X, Y$ tangent to $M$ the Gauss formula is given by

$$
\bar{\nabla}_{X} Y=\nabla_{X} Y+h(X, Y),
$$

where $h$ is the second fundamental form of $M$. If $D$ is the normal connection on the normal bundle of $M$, then for any normal vector field $\xi$ and any tangent vector field $X$ the Weingarten formula is given by

$$
\bar{\nabla}_{X} \xi=-A_{\xi} X+D_{X} \xi,
$$

where $A_{\xi}$ is the shape operator with respect to $\xi$. The shape operator and the second fundamental form are related by the formula

$$
\langle h(X, Y), \xi\rangle=\left\langle A_{\xi} X, Y\right\rangle
$$

for any $X$ and $Y$ tangent to $M$ and any $\xi$ normal to $M$.

The mean curvature vector field $H$ of $M$ in $\mathbb{E}_{2}^{4}$ is defined as

$$
H=\frac{1}{2} \operatorname{tr} h .
$$

Thus, if $M$ is a Lorentz surface and $\{X, Y\}$ is a local orthonormal frame of the tangent bundle such that $\langle X, X\rangle=1,\langle Y, Y\rangle=-1$, then the mean curvature vector field is given by the formula $H=\frac{1}{2}(h(X, X)-h(Y, Y))$. 
A surface $M$ is called minimal if its mean curvature vector vanishes identically, i.e. $H=0$. A surface $M$ is called quasi-minimal (or pseudo-minimal) if its mean curvature vector is lightlike at each point, i.e. $H \neq 0$ and $\langle H, H\rangle=0$. Obviously, quasi-minimal surfaces are always non-minimal.

$M$ is said to have constant mean curvature if $\langle H, H\rangle=$ const. We shall consider Lorentz surfaces in $\mathbb{E}_{2}^{4}$ for which $\langle H, H\rangle=$ const $\neq 0$. Such surfaces we call $C M C$ surfaces.

\section{Meridian Surfaces in Pseudo-Euclidean 4-Space}

Meridian surfaces in Euclidean 4-space we defined first in [12] as 2-dimensional surfaces lying on a standard rotational hypersurface in $\mathbb{E}^{4}$. These surfaces are one-parameter systems of meridians of the rotational hypersurface, that is why they are called meridian surfaces. The classification of meridian surfaces with constant Gauss curvature, with constant mean curvature, Chen meridian surfaces and meridian surfaces with parallel normal bundle is given in $\left[12\right.$ and [14. Meridian surfaces in $\mathbb{E}^{4}$ having pointwise 1-type Gauss map are classified in [1].

The idea from the Euclidean case is used in [13] for the construction of special families of two-dimensional spacelike surfaces lying on rotational hypersurfaces with timelike or spacelike axis in the Minkowski space $\mathbb{E}_{1}^{4}$. These surfaces are called meridian surface of elliptic or hyperbolic type, respectively. A local classification of marginally trapped meridian surfaces is given in [13. Meridian surfaces in $\mathbb{E}_{1}^{4}$ with pointwise 1-type Gauss map are classified in [2]. The classification of meridian surfaces of elliptic or hyperbolic type with constant Gauss curvature, with constant mean curvature, Chen meridian surfaces and meridian surfaces with parallel normal bundle is given in [15].

Following the idea from the Euclidean and Minkowski spaces, we shall construct Lorentz meridian surfaces in the pseudo-Euclidean 4 -space $\mathbb{E}_{2}^{4}$ as one-parameter systems of meridians of rotational hypersurfaces with timelike or spacelike axis.

3.1. Meridian surfaces lying on a rotational hypersurface with timelike axis. Let $O e_{1} e_{2} e_{3} e_{4}$ be the standard orthonormal frame in $\mathbb{E}_{2}^{4}$, i.e. $\left\langle e_{1}, e_{1}\right\rangle=\left\langle e_{2}, e_{2}\right\rangle=1,\left\langle e_{3}, e_{3}\right\rangle=$ $\left\langle e_{4}, e_{4}\right\rangle=-1$. First we consider a standard rotational hypersurface with timelike axis $O e_{4}$. Similarly, we can consider a rotational hypersurface with axis $O e_{3}$.

Since in the Minkowski space $\mathbb{E}_{1}^{3}=\operatorname{span}\left\{e_{1}, e_{2}, e_{3}\right\}$ there exist two types of spheres, namely the pseudo-sphere $\mathbb{S}_{1}^{2}(1)=\left\{V \in \mathbb{E}_{1}^{3}:\langle V, V\rangle=1\right\}$, i.e. the de Sitter space, and the pseudo-hyperbolic space $\mathbb{H}_{1}^{2}(-1)=\left\{V \in \mathbb{E}_{1}^{3}:\langle V, V\rangle=-1\right\}$, i.e. the anti-de Sitter space, we consider two types of rotational hypersurfaces about the axis $O e_{4}$.

\section{Rotational hypersurface of first type.}

Let $f=f(u), g=g(u)$ be smooth functions, defined in an interval $I \subset \mathbb{R}$. The first type rotational hypersurface $\mathcal{M}^{I}$ in $\mathbb{E}_{2}^{4}$, obtained by the rotation of the meridian curve $m: u \rightarrow(f(u), g(u))$ about the $O e_{4}$-axis, is parametrized as follows:

$$
\mathcal{M}^{I}: Z\left(u, w^{1}, w^{2}\right)=f(u)\left(\cosh w^{1} \cos w^{2} e_{1}+\cosh w^{1} \sin w^{2} e_{2}+\sinh w^{1} e_{3}\right)+g(u) e_{4} .
$$

If we denote by $l^{I}\left(w^{1}, w^{2}\right)=\cosh w^{1} \cos w^{2} e_{1}+\cosh w^{1} \sin w^{2} e_{2}+\sinh w^{1} e_{3}$ the unit position vector of the sphere $\mathbb{S}_{1}^{2}(1)$ in $\mathbb{E}_{1}^{3}$ centered at the origin $O$, then the parametrization of the rotational hypersurface $\mathcal{M}^{I}$ is written as:

$$
\mathcal{M}^{I}: Z\left(u, w^{1}, w^{2}\right)=f(u) l^{I}\left(w^{1}, w^{2}\right)+g(u) e_{4} .
$$

Now, we shall construct Lorentz surfaces in $\mathbb{E}_{2}^{4}$ which are one-parameter systems of meridians of the hypersurface $\mathcal{M}^{I}$. 
First we consider a smooth spacelike curve $c: l=l(v)=l^{I}\left(w^{1}(v), w^{2}(v)\right), v \in J, J \subset \mathbb{R}$ on $\mathbb{S}_{1}^{2}(1)$ parametrized by the arc-length, i.e. $\left\langle l^{\prime}, l^{\prime}\right\rangle=1$. We construct a two-dimensional surface $\mathcal{M}_{a}^{\prime}$ defined by:

$$
\mathcal{M}_{a}^{\prime}: z(u, v)=f(u) l(v)+g(u) e_{4}, \quad u \in I, v \in J
$$

Since the surface $\mathcal{M}_{a}^{\prime}$ is a one-parameter system of meridians of $\mathcal{M}^{I}$, we call it a meridian surface on $\mathcal{M}^{I}$.

The tangent space of $\mathcal{M}_{a}^{\prime}$ is spanned by the vector fields

$$
z_{u}=f^{\prime}(u) l(v)+g^{\prime}(u) e_{4} ; \quad z_{v}=f(u) l^{\prime}(v),
$$

so, the coefficients of the first fundamental form of $\mathcal{M}^{\prime}$ are

$$
E=\left\langle z_{u}, z_{u}\right\rangle=f^{\prime 2}-g^{\prime 2} ; \quad F=\left\langle z_{u}, z_{v}\right\rangle=0 ; \quad G=\left\langle z_{v}, z_{v}\right\rangle=f^{2}
$$

Since we are interested in Lorentz surfaces, we assume that the meridian curve $m$ is timelike, i.e. $f^{\prime 2}-g^{\prime 2}<0$. Without loss of generality we can assume that $f^{\prime 2}-g^{\prime 2}=-1$. Then the coefficients of the first fundamental form are $E=-1 ; F=0 ; G=f^{2}(u)$. We consider the unit tangent vector fields $X=z_{u}, Y=\frac{z_{v}}{f}=l^{\prime}$, which satisfy $\langle X, X\rangle=-1,\langle Y, Y\rangle=1$ and $\langle X, Y\rangle=0$.

Let $t(v)=l^{\prime}(v)$ be the tangent vector field of the curve $c$. Since $\langle t(v), t(v)\rangle=1$, $\langle l(v), l(v)\rangle=1$, and $\langle t(v), l(v)\rangle=0$, then there exists a unique (up to a sign) vector field $n(v)$, such that $\langle n(v), n(v)\rangle=-1$ and $\{l(v), t(v), n(v)\}$ is an orthonormal frame field in $\mathbb{E}_{1}^{3}=\operatorname{span}\left\{e_{1}, e_{2}, e_{3}\right\}$. With respect to this frame field we have the following Frenet formulas of $c$ on $\mathbb{S}_{1}^{2}(1)$ :

$$
\begin{aligned}
& l^{\prime}=t ; \\
& t^{\prime}=-\kappa n-l ; \\
& n^{\prime}=-\kappa t,
\end{aligned}
$$

where $\kappa(v)=\left\langle t^{\prime}(v), n(v)\right\rangle$ is the spherical curvature of $c$ on $\mathbb{S}_{1}^{2}(1)$. Now we consider the following normal vector fields:

$$
n_{1}=n(v) ; \quad n_{2}=g^{\prime}(u) l(v)+f^{\prime}(u) e_{4}
$$

which satisfy $\left\langle n_{1}, n_{1}\right\rangle=-1,\left\langle n_{2}, n_{2}\right\rangle=1,\left\langle n_{1}, n_{2}\right\rangle=0$.

Taking into account (2) and (3) we get the following derivative formulas:

$$
\begin{array}{lll}
\bar{\nabla}_{X} X=\kappa_{m} n_{2} ; & \bar{\nabla}_{X} n_{1}=0 ; \\
\bar{\nabla}_{X} Y=0 ; & \bar{\nabla}_{Y} n_{1}=-\frac{\kappa}{f} Y ; \\
\bar{\nabla}_{Y} X=\frac{f^{\prime}}{f} Y ; & \bar{\nabla}_{X} n_{2}=\kappa_{m} X ; \\
\bar{\nabla}_{Y} Y=\frac{f^{\prime}}{f} X-\frac{\kappa}{f} n_{1}-\frac{g^{\prime}}{f} n_{2} ; & \bar{\nabla}_{Y} n_{2}=\frac{g^{\prime}}{f} Y,
\end{array}
$$


where $\kappa_{m}$ denotes the curvature of the meridian curve $m$, which in case of a timelike curve is given by the formula $\kappa_{m}(u)=f^{\prime \prime}(u) g^{\prime}(u)-f^{\prime}(u) g^{\prime \prime}(u)$. Hence, we have

$$
\begin{aligned}
& h(X, X)=\kappa_{m} n_{2} ; \\
& h(X, Y)=0 ; \\
& h(Y, Y)=-\frac{\kappa}{f} n_{1}-\frac{g^{\prime}}{f} n_{2} .
\end{aligned}
$$

Formulas (5) imply that the mean curvature vector field $H$ of $\mathcal{M}_{a}^{\prime}$ is expressed as follows:

$$
H=-\frac{\kappa}{2 f} n_{1}-\frac{f \kappa_{m}+g^{\prime}}{2 f} n_{2}
$$

Using that $g^{\prime 2}=f^{\prime 2}+1$ and $\kappa_{m}=\frac{f^{\prime \prime}}{g^{\prime}}$, we obtain

$$
H=-\frac{\kappa}{2 f} n_{1}-\frac{f f^{\prime \prime}+\left(f^{\prime}\right)^{2}+1}{2 f \sqrt{f^{\prime 2}+1}} n_{2} .
$$

Now, let $c: l=l(v)=l^{I}\left(w^{1}(v), w^{2}(v)\right), v \in J, J \subset \mathbb{R}$ be a timelike curve on $\mathbb{S}_{1}^{2}(1)$ parametrized by the arc-length, i.e. $\left\langle l^{\prime}, l^{\prime}\right\rangle=-1$. We consider the two-dimensional surface $\mathcal{M}_{b}^{\prime}$ defined by:

$$
\mathcal{M}_{b}^{\prime}: z(u, v)=f(u) l(v)+g(u) e_{4}, \quad u \in I, v \in J,
$$

where $f^{\prime 2}-g^{\prime 2}=1$. The surface $\mathcal{M}_{b}^{\prime}$ is another meridian surface on $\mathcal{M}^{I}$.

In this case we consider an orthonormal frame field $\{l(v), t(v), n(v)\}$ of $\mathbb{E}_{1}^{3}$, such that $t=l^{\prime}$, $\langle l, l\rangle=1,\langle t, t\rangle=-1,\langle n, n\rangle=1$. Now, the Frenet formulas of $c$ on $\mathbb{S}_{1}^{2}(1)$ are:

$$
\begin{aligned}
& l^{\prime}=t ; \\
& t^{\prime}=\kappa n+l ; \\
& n^{\prime}=\kappa t,
\end{aligned}
$$

where $\kappa(v)=\left\langle t^{\prime}(v), n(v)\right\rangle$. The tangent vector fields of the meridian surface $\mathcal{M}_{b}^{\prime}$ are

$$
z_{u}=f^{\prime} l+g^{\prime} e_{4} ; \quad z_{v}=f t,
$$

and since $f^{\prime 2}-g^{\prime 2}=1$, the coefficients of the first fundamental form are $E=1 ; F=$ $0 ; G=-f^{2}(u)$. We consider the orthonormal tangent frame $X=z_{u}, Y=\frac{z_{v}}{f}=t$ and the orthonormal normal frame field defined by

$$
n_{1}=n ; \quad n_{2}=g^{\prime} l+f^{\prime} e_{4} .
$$

Thus, we obtain a frame field $\left\{X, Y, n_{1}, n_{2}\right\}$ of $\mathcal{M}_{b}^{\prime}$ such that $\left\langle n_{1}, n_{1}\right\rangle=1,\left\langle n_{2}, n_{2}\right\rangle=-1$ and $\left\langle n_{1}, n_{2}\right\rangle=0$. With respect to this frame field we have the following derivative formulas:

$$
\begin{array}{lll}
\bar{\nabla}_{X} X=\kappa_{m} n_{2} ; & \bar{\nabla}_{X} n_{1}=0 ; \\
\bar{\nabla}_{X} Y=0 ; & \bar{\nabla}_{Y} n_{1}=\frac{\kappa}{f} Y ; \\
\bar{\nabla}_{Y} X=\frac{f^{\prime}}{f} Y ; & \bar{\nabla}_{X} n_{2}=\kappa_{m} X ; \\
\bar{\nabla}_{Y} Y=\frac{f^{\prime}}{f} X+\frac{\kappa}{f} n_{1}-\frac{g^{\prime}}{f} n_{2} ; & \bar{\nabla}_{Y} n_{2}=\frac{g^{\prime}}{f} Y,
\end{array}
$$


where $\kappa_{m}$ is the curvature of the meridian curve $m$, which in the case of a spacelike curve is given by the formula $\kappa_{m}=f^{\prime} g^{\prime \prime}-f^{\prime \prime} g^{\prime}$.

Equalities (8) imply that the mean curvature vector field is given by the formula:

$$
H=-\frac{\kappa}{2 f} n_{1}+\frac{f \kappa_{m}+g^{\prime}}{2 f} n_{2}
$$

Having in mind that $g^{\prime 2}=f^{\prime 2}-1$ and $\kappa_{m}=\frac{f^{\prime \prime}}{g^{\prime}}$, we obtain

$$
H=-\frac{\kappa}{2 f} n_{1}+\frac{f f^{\prime \prime}+\left(f^{\prime}\right)^{2}-1}{2 f \sqrt{f^{\prime 2}-1}} n_{2} .
$$

So, we have two types of meridian surfaces lying on $\mathcal{M}^{I}$ : meridian surfaces of type $\mathcal{M}_{a}^{\prime}$ and meridian surfaces of type $\mathcal{M}_{b}^{\prime}$.

\section{Rotational hypersurface of second type.}

Now, we consider the second type rotational hypersurface $\mathcal{M}^{I I}$ in $\mathbb{E}_{2}^{4}$, obtained by the rotation of the meridian curve $m: u \rightarrow(f(u), g(u))$ about the axis $O e_{4}$, which is given by the following parametrization:

$$
\mathcal{M}^{I I}: Z\left(u, w^{1}, w^{2}\right)=f(u)\left(\sinh w^{1} \cos w^{2} e_{1}+\sinh w^{1} \sin w^{2} e_{2}+\cosh w^{1} e_{3}\right)+g(u) e_{4} .
$$

Note that $l^{I I}\left(w^{1}, w^{2}\right)=\sinh w^{1} \cos w^{2} e_{1}+\sinh w^{1} \sin w^{2} e_{2}+\cosh w^{1} e_{3}$ is the unit position vector of the hyperbolic sphere $\mathbb{H}_{1}^{2}(-1)$ in $\mathbb{E}_{1}^{3}=\operatorname{span}\left\{e_{1}, e_{2}, e_{3}\right\}$ centered at the origin $O$. So, the parametrization of $\mathcal{M}^{I I}$ can be written as:

$$
\mathcal{M}^{I I}: Z\left(u, w^{1}, w^{2}\right)=f(u) l^{I I}\left(w^{1}, w^{2}\right)+g(u) e_{4} .
$$

Meridian surfaces lying on the rotational hypersurface of second type $\mathcal{M}^{I I}$ can be constructed as follows. Let $c: l=l(v)=l^{I I}\left(w^{1}(v), w^{2}(v)\right)$ be a smooth curve on the hyperbolic sphere $\mathbb{H}_{1}^{2}(-1)$, where $w^{1}=w^{1}(v), w^{2}=w^{2}(v), v \in J, J \subset \mathbb{R}$. Then the two-dimensional surface $\mathcal{M}^{\prime \prime}$ defined by

$$
\mathcal{M}^{\prime \prime}: z(u, v)=f(u) l(v)+g(u) e_{4}, \quad u \in I, v \in J
$$

is a one-parameter system of meridians of $\mathcal{M}^{I I}$, which we call a meridian surface on $\mathcal{M}^{I I}$.

The tangent space of $\mathcal{M}^{\prime \prime}$ is spanned by the vector fields

$$
z_{u}=f^{\prime}(u) l(v)+g^{\prime}(u) e_{4} ; \quad z_{v}=f(u) l^{\prime}(v),
$$

so the coefficients of the first fundamental form of $\mathcal{M}^{\prime \prime}$ are

$$
E=\left\langle z_{u}, z_{u}\right\rangle=-\left(f^{\prime 2}+g^{\prime 2}\right) ; \quad F=\left\langle z_{u}, z_{v}\right\rangle=0 ; \quad G=\left\langle z_{v}, z_{v}\right\rangle=f^{2}\left\langle l^{\prime}, l^{\prime}\right\rangle .
$$

Since $c$ is a curve lying on $\mathbb{H}_{1}^{2}(-1)$, we have $\langle l, l\rangle=-1$, which implies that the tangent vector field $t=l^{\prime}$ satisfies $\langle t, t\rangle=1$. Without loss of generality we suppose that $f^{\prime 2}+g^{\prime 2}=1$. Then the coefficients of the first fundamental form of $\mathcal{M}^{\prime \prime}$ are $E=-1 ; F=0 ; G=f^{2}$.

We consider an orthonormal frame field $\{l(v), t(v), n(v)\}$ of $c$ satisfying the conditions $\langle l, l\rangle=-1,\langle t, t\rangle=1,\langle n, n\rangle=1$. The Frenet formulas of $c$ on $\mathbb{H}_{1}^{2}(-1)$ are:

$$
\begin{aligned}
& l^{\prime}=t ; \\
& t^{\prime}=\kappa n+l ; \\
& n^{\prime}=-\kappa t,
\end{aligned}
$$

where $\kappa(v)=\left\langle t^{\prime}(v), n(v)\right\rangle$ is the curvature of $c$ on $\mathbb{H}_{1}^{2}(-1)$. 
Let us consider the following orthonormal frame field of $\mathcal{M}^{\prime \prime}$ :

$$
X=z_{u} ; \quad Y=\frac{z_{v}}{f}=t ; \quad n_{1}=n(v) ; \quad n_{2}=-g^{\prime}(u) l(v)+f^{\prime}(u) e_{4} .
$$

This frame field satisfies $\langle X, X\rangle=-1,\langle Y, Y\rangle=1,\langle X, Y\rangle=0,\left\langle n_{1}, n_{1}\right\rangle=1,\left\langle n_{2}, n_{2}\right\rangle=-1$, $\left\langle n_{1}, n_{2}\right\rangle=0$. Taking into account (11) we get the following derivative formulas:

$$
\begin{array}{lll}
\bar{\nabla}_{X} X=\kappa_{m} n_{2} ; & \bar{\nabla}_{X} n_{1}=0 ; \\
\bar{\nabla}_{X} Y=0 ; & \bar{\nabla}_{Y} n_{1}=-\frac{\kappa}{f} Y ; \\
\bar{\nabla}_{Y} X=\frac{f^{\prime}}{f} Y ; & \bar{\nabla}_{X} n_{2}=-\kappa_{m} X ; \\
\bar{\nabla}_{Y} Y=\frac{f^{\prime}}{f} X+\frac{\kappa}{f} n_{1}-\frac{g^{\prime}}{f} n_{2} ; & \bar{\nabla}_{Y} n_{2}=-\frac{g^{\prime}}{f} Y,
\end{array}
$$

where $\kappa_{m}=f^{\prime} g^{\prime \prime}-f^{\prime \prime} g^{\prime}$. Hence, we obtain the formulas

$$
\begin{aligned}
& h(X, X)=\kappa_{m} n_{2} ; \\
& h(X, Y)=0 ; \\
& h(Y, Y)=\frac{\kappa}{f} n_{1}-\frac{g^{\prime}}{f} n_{2},
\end{aligned}
$$

which imply that the normal mean curvature vector field of $\mathcal{M}^{\prime \prime}$ is given by

$$
H=\frac{\kappa}{2 f} n_{1}+\frac{f f^{\prime \prime}+\left(f^{\prime}\right)^{2}-1}{2 f \sqrt{1-f^{\prime 2}}} n_{2} .
$$

Note that we can construct only one type of Lorentz meridian surfaces lying on the rotational hypersurface $\mathcal{M}^{I I}$.

3.2. Meridian surfaces lying on a rotational hypersurface with spacelike axis. In a similar way, we can construct meridian surfaces lying on a rotational hypersurface with spacelike axis $O e_{1}$ (or $\left.O e_{2}\right)$.

In the Minkowski space $\mathbb{E}_{2}^{3}=\operatorname{span}\left\{e_{2}, e_{3}, e_{4}\right\}$ there exist two types of spheres, namely the de Sitter space $\mathbb{S}_{2}^{2}(1)=\left\{V \in \mathbb{E}_{2}^{3}:\langle V, V\rangle=1\right\}$, and the hyperbolic space $\mathbb{H}_{1}^{2}(-1)=$ $\left\{V \in \mathbb{E}_{2}^{3}:\langle V, V\rangle=-1\right\}$. So, we can consider two types of rotational hypersurfaces about the axis $O e_{1}$.

\section{Rotational hypersurface of first type.}

Let $f=f(u), g=g(u)$ be smooth functions, defined in an interval $I \subset \mathbb{R}$. We denote by $\tilde{l}^{I}\left(w^{1}, w^{2}\right)=\cosh w^{1} e_{2}+\sinh w^{1} \cos w^{2} e_{3}+\sinh w^{1} \sin w^{2} e_{4}$ the unit position vector of the sphere $\mathbb{S}_{2}^{2}(1)$ in $\mathbb{E}_{2}^{3}=\operatorname{span}\left\{e_{2}, e_{3}, e_{4}\right\}$ centered at the origin $O$. Then, the first type rotational hypersurface $\widetilde{\mathcal{M}}^{I}$, obtained by the rotation of the meridian curve $m: u \rightarrow(f(u), g(u))$ about the axis $O e_{1}$, is parametrized by

$$
\widetilde{\mathcal{M}}^{I}: Z\left(u, w^{1}, w^{2}\right)=g(u) e_{1}+f(u)\left(\cosh w^{1} e_{2}+\sinh w^{1} \cos w^{2} e_{3}+\sinh w^{1} \sin w^{2} e_{4}\right),
$$

or equivalently,

$$
\widetilde{\mathcal{M}}^{I}: Z\left(u, w^{1}, w^{2}\right)=g(u) e_{1}+f(u) \tilde{l}^{I}\left(w^{1}, w^{2}\right) .
$$

Lorentz meridian surfaces lying on $\widetilde{\mathcal{M}}^{I}$ are one-parameter systems of meridians of $\widetilde{\mathcal{M}}^{I}$. They can be constructed as follows. Let $c: l=l(v)=\tilde{l}^{I}\left(w^{1}(v), w^{2}(v)\right), v \in J, J \subset \mathbb{R}$ be a 
smooth curve on $\mathbb{S}_{2}^{2}(1)$. We consider the two-dimensional surface $\widetilde{\mathcal{M}^{\prime}}$ defined by:

$$
\widetilde{\mathcal{M}^{\prime}}: z(u, v)=g(u) e_{1}+f(u) l(v), \quad u \in I, v \in J .
$$

It is a one-parameter system of meridians of $\widetilde{\mathcal{M}}^{I}$, so we call $\widetilde{\mathcal{M}}^{\prime}$ a meridian surface on $\widetilde{\mathcal{M}}^{I}$.

It can easily be seen that the meridian surface $\mathcal{M}^{\prime \prime}$, defined by (10), can be transformed into the surface $\widetilde{\mathcal{M}^{\prime}}$ by the transformation $T$ given by the matrix

$$
T=\left(\begin{array}{llll}
0 & 0 & 0 & 1 \\
0 & 0 & 1 & 0 \\
1 & 0 & 0 & 0 \\
0 & 1 & 0 & 0
\end{array}\right)
$$

So, the meridian surface $\mathcal{M}^{\prime \prime}$ lying on $\mathcal{M}^{I I}$ and the meridian surface $\widetilde{\mathcal{M}}^{\prime}$ lying on $\widetilde{\mathcal{M}}^{I}$ are congruent. Hence, all results concerning the surface $\mathcal{M}^{\prime \prime}$ hold true for the surface $\widetilde{\mathcal{M}^{\prime}}$.

\section{Rotational hypersurface of second type.}

The second type rotational hypersurface $\widetilde{\mathcal{M}}^{I I}$, obtained by the rotation of the meridian curve $m$ about $O e_{1}$, is given by the following parametrization:

$$
\widetilde{\mathcal{M}}^{I I}: Z\left(u, w^{1}, w^{2}\right)=g(u) e_{1}+f(u)\left(\sinh w^{1} e_{2}+\cosh w^{1} \cos w^{2} e_{3}+\cosh w^{1} \sin w^{2} e_{4}\right) .
$$

Here, $\tilde{l}^{I I}\left(w^{1}, w^{2}\right)=\sinh w^{1} e_{2}+\cosh w^{1} \cos w^{2} e_{3}+\cosh w^{1} \sin w^{2} e_{4}$ is the unit position vector of the hyperbolic sphere $\mathbb{H}_{1}^{2}(-1)$ in $\mathbb{E}_{2}^{3}=\operatorname{span}\left\{e_{2}, e_{3}, e_{4}\right\}$ centered at the origin $O$. So, the parametrization of $\widetilde{\mathcal{M}}^{I I}$ can be written as

$$
\widetilde{\mathcal{M}}^{I I}: Z\left(u, w^{1}, w^{2}\right)=g(u) e_{1}+f(u) \tilde{l}^{I I}\left(w^{1}, w^{2}\right) .
$$

We can construct two types of meridian surfaces lying on the second type rotational hypersurface $\widetilde{\mathcal{M}}^{I I}$.

First, we consider a smooth spacelike curve $c: l=l(v)=\tilde{l}^{I I}\left(w^{1}(v), w^{2}(v)\right), v \in J, J \subset \mathbb{R}$ lying on the hyperbolic sphere $\mathbb{H}_{1}^{2}(-1)$ in $\mathbb{E}_{2}^{3}$. We assume that $c$ is parametrized by the arc-length, i.e. $\left\langle l^{\prime}, l^{\prime}\right\rangle=1$. Let $\widetilde{\mathcal{M}}_{a}^{\prime \prime}$ be the surface lying on $\widetilde{\mathcal{M}}^{I I}$ and defined by:

$$
\widetilde{\mathcal{M}_{a}^{\prime \prime}}: z(u, v)=g(u) e_{1}+f(u) l(v), \quad u \in I, v \in J .
$$

The tangent space of $\widetilde{\mathcal{M}}_{a}^{\prime \prime}$ is spanned by the vector fields

$$
z_{u}=g^{\prime}(u) e_{1}+f^{\prime}(u) l(v) ; \quad z_{v}=f(u) l^{\prime}(v),
$$

so, the coefficients of the first fundamental form are

$$
E=g^{\prime 2}-f^{\prime 2} ; \quad F=0 ; \quad G=f^{2} .
$$

Since we are interested in Lorentz surfaces, we assume that $f^{\prime 2}-g^{\prime 2}=1$. Then the coefficients of the first fundamental form are $E=-1 ; F=0 ; G=f^{2}$. It is easy to see that under the tranformation $T$ given by (12) the surface $\mathcal{M}_{b}^{\prime}$ is transformed into the surface $\widetilde{\mathcal{M}}_{a}^{\prime \prime}$. So, all results concerning the surface $\mathcal{M}_{b}^{\prime}$ hold true for the surface $\widetilde{\mathcal{M}}_{a}^{\prime \prime}$.

Second, we consider a timelike curve $c: l=l(v)=\tilde{l}^{I I}\left(w^{1}(v), w^{2}(v)\right), v \in J, J \subset \mathbb{R}$ lying on the hyperbolic sphere $\mathbb{H}_{1}^{2}(-1)$ and parametrized by the arc-length, i.e. $\left\langle l^{\prime}, l^{\prime}\right\rangle=-1$. Then the surface $\widetilde{\mathcal{M}}_{b}^{\prime \prime}$ defined by:

$$
\widetilde{\mathcal{M}_{b}^{\prime \prime}}: z(u, v)=g(u) e_{1}+f(u) l(v), \quad u \in I, v \in J
$$


where $f^{\prime 2}-g^{\prime 2}=-1$ is a Lorentz meridian surface lying on $\widetilde{\mathcal{M}}^{I I}$. It is clear that the meridian surfaces $\mathcal{M}_{a}^{\prime}$ and $\widetilde{\mathcal{M}}_{b}^{\prime \prime}$ are congruent up to the transformation $T$.

So, it is worth studying three types of Lorentz meridian surfaces in $\mathbb{E}_{2}^{4}$, namely the surfaces denoted by $\mathcal{M}_{a}^{\prime}, \mathcal{M}_{b}^{\prime}$, and $\mathcal{M}^{\prime \prime}$.

\section{Minimal Meridian Surfaces in $\mathbb{E}_{2}^{4}$}

In this section we give the classification of all minimal meridian surfaces in $\mathbb{E}_{2}^{4}$.

Theorem 1. Let $\mathcal{M}_{a}^{\prime}$ be a meridian surface on $\mathcal{M}^{I}$ defined by (1). Then $\mathcal{M}_{a}^{\prime}$ is minimal if and only if the curve $c$ has zero spherical curvature and the meridian curve $m$ is given by

$$
f(u)= \pm \sqrt{-u^{2}+2 a u+b}, \quad g(u)= \pm \sqrt{a^{2}+b} \arcsin \frac{u-a}{\sqrt{a^{2}+b}}+c,
$$

where $a=$ const, $b=$ const, $c=$ const.

Proof. The mean curvature vector field $H$ of the meridian surface $\mathcal{M}_{a}^{\prime}$ is given by formula (6). Hence, $\mathcal{M}_{a}^{\prime}$ is minimal if and only if the curvature of $c$ is $\kappa=0$ and the function $f(u)$ satisfies the following equation

$$
f f^{\prime \prime}+\left(f^{\prime}\right)^{2}+1=0
$$

The solutions of this differential equation are given by the formula $f(u)= \pm \sqrt{-u^{2}+2 a u+b}$, where $a=$ const, $b=$ const. Having in mind that $g^{\prime}=\sqrt{f^{\prime 2}+1}$, we get the following equation for $g(u)$ :

$$
g^{\prime}= \pm \frac{\sqrt{a^{2}+b}}{\sqrt{-u^{2}+2 a u+b}} .
$$

Integrating the above equation we obtain

$$
g(u)= \pm \sqrt{a^{2}+b} \arcsin \frac{u-a}{\sqrt{a^{2}+b}}+c, \quad c=\text { const } .
$$

Note that if $\mathcal{M}_{a}^{\prime}$ is minimal, then $\kappa=0$ and from (41) we get that $\bar{\nabla}_{X} n_{1}=0, \bar{\nabla}_{Y} n_{1}=0$. This means that the normal vector field $n_{1}$ is constant. Hence, the surface $\mathcal{M}_{a}^{\prime}$ lies in the constant 3-dimensional space $\mathbb{E}_{1}^{3}=\operatorname{span}\left\{X, Y, n_{2}\right\}$. Consequently, $\mathcal{M}_{a}^{\prime}$ lies in a hyperplane of $\mathbb{E}_{2}^{4}$.

Theorem 2. Let $\mathcal{M}_{b}^{\prime}$ be a meridian surface on $\mathcal{M}^{I}$ defined by (7). Then $\mathcal{M}_{b}^{\prime}$ is minimal if and only if the curve $c$ has zero spherical curvature and the meridian curve $m$ is given by

$$
f(u)= \pm \sqrt{u^{2}+2 a u+b}, \quad g(u)= \pm \sqrt{a^{2}-b} \ln \left|u+a+\sqrt{u^{2}+2 a u+b}\right|+c,
$$

where $a=$ const, $b=$ const, $c=$ const, $a^{2}-b>0$.

Proof. Using that the mean curvature vector field $H$ of $\mathcal{M}_{b}^{\prime}$ is given by formula (9), we get that $\mathcal{M}_{b}^{\prime}$ is minimal if and only if $\kappa=0$ and the function $f(u)$ satisfies the equation

$$
f f^{\prime \prime}+\left(f^{\prime}\right)^{2}-1=0 .
$$

The solutions of this differential equation are given by the formula $f(u)= \pm \sqrt{u^{2}+2 a u+b}$, where $a=$ const, $b=$ const. Using that $g^{\prime}=\sqrt{f^{\prime 2}-1}$, we get the following equation for $g(u)$ :

$$
g^{\prime}= \pm \frac{\sqrt{a^{2}-b}}{\sqrt{u^{2}+2 a u+b}}
$$


Integrating the last equation we obtain

$$
g(u)= \pm \sqrt{a^{2}-b} \ln \left|u+a+\sqrt{u^{2}+2 a u+b}\right|+c, \quad c=\text { const } .
$$

Note that if $\mathcal{M}_{b}^{\prime}$ is minimal, we have $\kappa=0$ and again we obtain $\bar{\nabla}_{X} n_{1}=0, \bar{\nabla}_{Y} n_{1}=0$, i.e. the normal vector field $n_{1}$ is constant. In this case the surface $\mathcal{M}_{b}^{\prime}$ lies in the constant 3-dimensional space $\mathbb{E}_{2}^{3}=\operatorname{span}\left\{X, Y, n_{2}\right\}$. Consequently, $\mathcal{M}_{b}^{\prime}$ lies in a hyperplane of $\mathbb{E}_{2}^{4}$.

Theorem 3. Let $\mathcal{M}^{\prime \prime}$ be a meridian surface on $\mathcal{M}^{I I}$ defined by (10). Then $\mathcal{M}^{\prime \prime}$ is minimal if and only if the curve $c$ has zero spherical curvature and the meridian curve $m$ is given by

$$
f(u)= \pm \sqrt{u^{2}+2 a u+b}, \quad g(u)= \pm \sqrt{b-a^{2}} \ln \left|u+a+\sqrt{u^{2}+2 a u+b}\right|+c,
$$

where $a=$ const, $b=$ const, $c=$ const, $b-a^{2}>0$.

The proof is similar to the proof of the previous two theorems. Again we have that if $\mathcal{M}^{\prime \prime}$ is minimal, then it lies in a constant 3-dimensional hyperplane $\mathbb{E}_{2}^{3}$ of $\mathbb{E}_{2}^{4}$.

Finally, we can formulate the following

Corollary 4. There are no minimal meridian surfaces lying fully in $\mathbb{E}_{2}^{4}$.

\section{Quasi-Minimal Meridian Surfaces in $\mathbb{E}_{2}^{4}$}

In this section we classify all quasi-minimal meridian surfaces in the pseudo-Euclidean 4-space $\mathbb{E}_{2}^{4}$.

Theorem 5. Let $\mathcal{M}_{a}^{\prime}$ be a meridian surface on $\mathcal{M}^{I}$ defined by (11). Then $\mathcal{M}_{a}^{\prime}$ is quasiminimal if and only if the curve $c$ has constant curvature $\kappa=a=$ const, $a \neq 0$ and the meridian curve $m$ is determined by $f^{\prime}=\varphi(f)$ where

$$
\varphi(t)= \pm \frac{1}{t} \sqrt{( \pm a t+c)^{2}-t^{2}}, \quad c=\text { const }
$$

$g(u)$ is defined by $g^{\prime}=\sqrt{f^{\prime 2}+1}$.

Proof. Using formula (6) for the mean curvature vector field $H$ of the meridian surface $\mathcal{M}_{a}^{\prime}$, we get that $\mathcal{M}_{a}^{\prime}$ is quasi-minimal, i.e. $H \neq 0$ and $\langle H, H\rangle=0$, if and only if

$$
\frac{\left(f f^{\prime \prime}+\left(f^{\prime}\right)^{2}+1\right)^{2}}{f^{\prime 2}+1}=\kappa^{2}, \quad \kappa \neq 0
$$

The left-hand side of this equation is a function of $u$, the right-hand side of the equation is a function of $v$. Hence, we obtain

$$
\begin{aligned}
& \kappa=a, \quad a=\text { const } \neq 0 \\
& \left(f f^{\prime \prime}+\left(f^{\prime}\right)^{2}+1\right)^{2}=a^{2}\left(f^{\prime 2}+1\right) .
\end{aligned}
$$

So, the meridian $m$ is determined by the following differential equation:

$$
f f^{\prime \prime}+\left(f^{\prime}\right)^{2}+1= \pm a \sqrt{f^{\prime 2}+1}
$$

The solutions of the above differential equation can be found in the following way. If we set $f^{\prime}=\varphi(f)$ in equation (13), we obtain that the function $\varphi=\varphi(t)$ is a solution of the equation:

$$
\frac{t}{2}\left(\varphi^{2}\right)^{\prime}+\varphi^{2}+1= \pm a \sqrt{\varphi^{2}+1}
$$


Now, we set $z(t)=\sqrt{\varphi^{2}(t)+1}$, so, equation (14) takes the form

$$
z^{\prime}+\frac{1}{t} z= \pm \frac{a}{t}
$$

The general solution of the last equation is given by the formula $z(t)=\frac{ \pm a t+c}{t}, c=$ const. Hence, the general solution of (14) is

$$
\varphi(t)= \pm \frac{1}{t} \sqrt{( \pm a t+c)^{2}-t^{2}}
$$

Similarly to the proof of Theorem 5 we obtain the classification of all quasi-minimal meridian surface of type $\mathcal{M}_{b}^{\prime}$.

Theorem 6. Let $\mathcal{M}_{b}^{\prime}$ be a meridian surface on $\mathcal{M}^{I}$ defined by (77). Then $\mathcal{M}_{b}^{\prime}$ is quasiminimal if and only if the curve $c$ has constant curvature $\kappa=a=$ const, $a \neq 0$ and the meridian curve $m$ is determined by $f^{\prime}=\varphi(f)$ where

$$
\varphi(t)= \pm \frac{1}{t} \sqrt{(c \pm a t)^{2}+t^{2}}, \quad c=\text { const }
$$

$g(u)$ is defined by $g^{\prime}=\sqrt{f^{\prime 2}-1}$.

The classification of all quasi-minimal meridian surface of type $\mathcal{M}^{\prime \prime}$ is given in the next theorem.

Theorem 7. Let $\mathcal{M}^{\prime \prime}$ be a meridian surface on $\mathcal{M}^{I I}$ defined by (10). Then $\mathcal{M}^{\prime \prime}$ is quasiminimal if and only if the curve $c$ has constant curvature $\kappa=a=$ const, $a \neq 0$ and the meridian curve $m$ is determined by $f^{\prime}=\varphi(f)$ where

$$
\varphi(t)= \pm \frac{1}{t} \sqrt{t^{2}-(c \pm a t)^{2}}, \quad c=\text { const }
$$

$g(u)$ is defined by $g^{\prime}=\sqrt{1-f^{\prime 2}}$.

The proof is similar to the proof of Theorem 5 .

\section{Meridian Surfaces with Constant Mean Curvature in $\mathbb{E}_{2}^{4}$}

In this section we shall classify all meridian surfaces with non-zero constant mean curvature.

Let $\mathcal{M}_{a}^{\prime}$ be a meridian surface on $\mathcal{M}^{I}$ defined by (1). The mean curvature vector field $H$ of $\mathcal{M}_{a}^{\prime}$ is given by formula (6). So, we have

$$
\langle H, H\rangle=\frac{\left(f f^{\prime \prime}+\left(f^{\prime}\right)^{2}+1\right)^{2}-\kappa^{2}\left(f^{\prime 2}+1\right)}{4 f^{2}\left(f^{\prime 2}+1\right)} .
$$

In the next theorem we classify the meridian surfaces of type $\mathcal{M}_{a}^{\prime}$ for which $\langle H, H\rangle=c=$ const, $c \neq 0$.

Theorem 8. Let $\mathcal{M}_{a}^{\prime}$ be a meridian surface on $\mathcal{M}^{I}$ defined by (11). Then $\mathcal{M}_{a}^{\prime}$ has nonzero constant mean curvature, i.e. $\langle H, H\rangle=c=$ const, $c \neq 0$, if and only if the curve $c$ 
has constant curvature $\kappa=a=$ const, $a \neq 0$ and the meridian curve $m$ is determined by $f^{\prime}=\varphi(f)$ where

$$
\begin{aligned}
& \varphi(t)= \pm \frac{1}{t} \sqrt{\left(b \pm \frac{t}{2} \sqrt{a^{2}+4 c t^{2}} \pm \frac{a^{2}}{4 \sqrt{c}} \ln \left|2 \sqrt{c} t+\sqrt{a^{2}+4 c t^{2}}\right|\right)^{2}-t^{2}}, \quad \text { if } c>0, \\
& \varphi(t)= \pm \frac{1}{t} \sqrt{\left(b \pm \frac{t}{2} \sqrt{a^{2}-4 c t^{2}} \pm \frac{a^{2}}{4 \sqrt{-c}} \arcsin \frac{2 \sqrt{-c} t}{a}\right)^{2}-t^{2}, \quad \text { if } c<0,}
\end{aligned}
$$

$b=$ const, and $g(u)$ is defined by $g^{\prime}=\sqrt{f^{\prime 2}+1}$.

Proof. Let $\mathcal{M}_{a}^{\prime}$ be a meridian surface on $\mathcal{M}^{I}$. It follows from (15) that the condition on $\mathcal{M}_{a}^{\prime}$ to have non-zero constant mean curvature, i.e. $\langle H, H\rangle=c=$ const, $c \neq 0$ is equivalent to the equality

$$
\frac{\left(f f^{\prime \prime}+\left(f^{\prime}\right)^{2}+1\right)^{2}-\kappa^{2}\left(f^{\prime 2}+1\right)}{4 f^{2}\left(f^{\prime 2}+1\right)}=c .
$$

The last equality can be written as

$$
\frac{\left(f f^{\prime \prime}+\left(f^{\prime}\right)^{2}+1\right)^{2}-4 c f^{2}\left(f^{\prime 2}+1\right)}{f^{\prime 2}+1}=\kappa^{2}
$$

Since the left-hand side of (16) is a function of $u$, the right-hand side of (16) is a function of $v$, we obtain

$$
\begin{aligned}
& \kappa=a, \quad a=\text { const } \neq 0 \\
& \frac{\left(f f^{\prime \prime}+\left(f^{\prime}\right)^{2}+1\right)^{2}-4 c f^{2}\left(f^{\prime 2}+1\right)}{f^{\prime 2}+1}=a^{2} .
\end{aligned}
$$

Hence, the meridian $m$ is determined by the solutions of the following differential equation:

$$
\left(f f^{\prime \prime}+\left(f^{\prime}\right)^{2}+1\right)^{2}-4 c f^{2}\left(f^{\prime 2}+1\right)=a^{2}\left(f^{\prime 2}+1\right) .
$$

We can find the solutions of the last equation by setting $f^{\prime}=\varphi(f)$. Then we obtain that the function $\varphi=\varphi(t)$ is a solution of the equation:

$$
\left(\frac{t}{2}\left(\varphi^{2}\right)^{\prime}+\varphi^{2}+1\right)^{2}-4 c t^{2}\left(\varphi^{2}+1\right)=a^{2}\left(\varphi^{2}+1\right) .
$$

If we set $z(t)=\sqrt{\varphi^{2}(t)+1}$, from equation (17) we get

$$
z^{\prime}+\frac{1}{t} z= \pm \frac{\sqrt{a^{2}+4 c t^{2}}}{t}
$$

The general solution of the last equation is given by the formula $z(t)=\frac{1}{t}\left(b \pm \int \sqrt{a^{2}+4 c t^{2}} d t\right)$, where $b=$ const.

If $c>0$, i.e. the mean curvature vector field $H$ is spacelike, then $\int \sqrt{a^{2}+4 c t^{2}} d t=$ $\frac{t}{2} \sqrt{a^{2}+4 c t^{2}}+\frac{a^{2}}{4 \sqrt{c}} \ln \left|2 \sqrt{c} t+\sqrt{a^{2}+4 c t^{2}}\right|$. Hence,

$$
z(t)=\frac{1}{t}\left(b \pm \frac{t}{2} \sqrt{a^{2}+4 c t^{2}} \pm \frac{a^{2}}{4 \sqrt{c}} \ln \left|2 \sqrt{c} t+\sqrt{a^{2}+4 c t^{2}}\right|\right)
$$


and the general solution of (17) is

$$
\varphi(t)= \pm \frac{1}{t} \sqrt{\left(b \pm \frac{t}{2} \sqrt{a^{2}+4 c t^{2}} \pm \frac{a^{2}}{4 \sqrt{c}} \ln \left|2 \sqrt{c} t+\sqrt{a^{2}+4 c t^{2}}\right|\right)^{2}-t^{2}} .
$$

If $c<0$, i.e. the mean curvature vector field $H$ is timelike, then $\int \sqrt{a^{2}+4 c t^{2}} d t=$ $\frac{t}{2} \sqrt{a^{2}-4 c t^{2}}+\frac{a^{2}}{4 \sqrt{-c}} \arcsin \frac{2 \sqrt{-c} t}{a}$. Hence, the function $z(t)$ is given by

$$
z(t)=\frac{1}{t}\left(b \pm \frac{t}{2} \sqrt{a^{2}-4 c t^{2}} \pm \frac{a^{2}}{4 \sqrt{-c}} \arcsin \frac{2 \sqrt{-c} t}{a}\right)
$$

and the general solution of (17) is

$$
\varphi(t)= \pm \frac{1}{t} \sqrt{\left(b \pm \frac{t}{2} \sqrt{a^{2}-4 c t^{2}} \pm \frac{a^{2}}{4 \sqrt{-c}} \arcsin \frac{2 \sqrt{-c} t}{a}\right)^{2}-t^{2}}
$$

Now, let $\mathcal{M}_{b}^{\prime}$ be a meridian surface on $\mathcal{M}^{I}$ defined by (7). Hence, the mean curvature vector field $H$ of $\mathcal{M}_{b}^{\prime}$ is given by formula (9) and

$$
\langle H, H\rangle=\frac{\kappa^{2}\left(f^{\prime 2}-1\right)-\left(f f^{\prime \prime}+\left(f^{\prime}\right)^{2}-1\right)^{2}}{4 f^{2}\left(f^{\prime 2}-1\right)} .
$$

The classification of the meridian surfaces of type $\mathcal{M}_{b}^{\prime}$ for which $\langle H, H\rangle=c=$ const, $c \neq 0$ is given in the following theorem.

Theorem 9. Let $\mathcal{M}_{b}^{\prime}$ be a meridian surface on $\mathcal{M}^{I}$ defined by (7). Then $\mathcal{M}_{b}^{\prime}$ has nonzero constant mean curvature, i.e. $\langle H, H\rangle=c=$ const, $c \neq 0$, if and only if the curve $c$ has constant curvature $\kappa=a=$ const, $a \neq 0$ and the meridian curve $m$ is determined by $f^{\prime}=\varphi(f)$ where

$$
\begin{aligned}
& \varphi(t)= \pm \frac{1}{t} \sqrt{\left(b \pm \frac{t}{2} \sqrt{a^{2}-4 c t^{2}} \pm \frac{a^{2}}{4 \sqrt{c}} \arcsin \frac{2 \sqrt{c} t}{a}\right)^{2}+t^{2}, \quad \text { if } c>0,} \\
& \varphi(t)= \pm \frac{1}{t} \sqrt{\left(b \pm \frac{t}{2} \sqrt{a^{2}-4 c t^{2}} \pm \frac{a^{2}}{4 \sqrt{-c}} \ln \left|2 \sqrt{-c} t+\sqrt{a^{2}-4 c t^{2}}\right|\right)^{2}+t^{2}, \quad \text { if } c<0},
\end{aligned}
$$

$b=$ const, and $g(u)$ is defined by $g^{\prime}=\sqrt{f^{\prime 2}-1}$.

The proof of this theorem is similar to the proof of Theorem 8

At the end of this section we give the classification of the meridian surfaces of type $\mathcal{M}^{\prime \prime}$ for which $\langle H, H\rangle=c=$ const, $c \neq 0$.

Theorem 10. Let $\mathcal{M}^{\prime \prime}$ be a meridian surface on $\mathcal{M}^{I I}$ defined by (10). Then $\mathcal{M}^{\prime \prime}$ has nonzero constant mean curvature, i.e. $\langle H, H\rangle=c=$ const, $c \neq 0$, if and only if the curve $c$ has constant curvature $\kappa=a=$ const, $a \neq 0$ and the meridian curve $m$ is determined by 
$f^{\prime}=\varphi(f)$ where

$$
\begin{aligned}
& \varphi(t)=\frac{1}{t} \sqrt{t^{2}-\left(b \mp \frac{t}{2} \sqrt{a^{2}-4 c t^{2}} \mp \frac{a^{2}}{4 \sqrt{c}} \arcsin \frac{2 \sqrt{c} t}{a}\right)^{2}}, \quad \text { if } c>0, \\
& \varphi(t)= \pm \frac{1}{t} \sqrt{t^{2}-\left(b \mp \frac{t}{2} \sqrt{a^{2}-4 c t^{2}} \mp \frac{a^{2}}{4 \sqrt{-c}} \ln \left|2 \sqrt{-c} t+\sqrt{a^{2}-4 c t^{2}}\right|\right)^{2}}, \quad \text { if } c<0,
\end{aligned}
$$

$b=$ const, and $g(u)$ is defined by $g^{\prime}=\sqrt{1-f^{\prime 2}}$.

Acknowledgments: The second author is partially supported by the Bulgarian National Science Fund, Ministry of Education and Science of Bulgaria under contract DFNI-I 02/14.

The paper is prepared during the first author's visit at the Institute of Mathematics and Informatics at the Bulgarian Academy of Sciences, Sofia, Bulgaria in November - December 2015.

\section{REFERENCES}

[1] Arslan K., Bulca B., Milousheva V., Meridian surfaces in $\mathbb{E}^{4}$ with pointwise 1-type Gauss map, Bull. Korean Math. Soc., 51, no. 3 (2014), 911-922.

[2] Arslan K., Milousheva V., Meridian surfaces of elliptic or hyperbolic type with pointwise 1-type Gauss map in Minkowski 4-space, Taiwanese J. Math., 20, no. 2 (2016), 311-332.

[3] Brander D., Singularities of spacelike constant mean curvature surfaces in Lorentz-Minkowski space. Math. Proc. Camb. Phil. Soc. 150 (2011), 527-556.

[4] Chaves R., Cândido, C., The Gauss map of spacelike rotational surfaces with constant mean curvature in the Lorentz-Minkowski space. Differential geometry, Valencia, 2001, 106-114, World Sci. Publ., River Edge, NJ, 2002.

[5] Chen B.-Y., Classification of marginally trapped Lorentzian flat surfaces in $\mathbb{E}_{2}^{4}$ and its application to biharmonic surfaces, J. Math. Anal. Appl., 2008, 340(2), 861-875.

[6] Chen B.-Y., Classification of marginally trapped surfaces of constant curvature in Lorentzian complex plane, Hokkaido Math. J., 2009, 38(2), 361-408.

[7] Chen B.-Y., Black holes, marginally trapped surfaces and quasi-minimal surfaces, Tamkang J. Math., 2009, 40(4), 313-341.

[8] Chen B.-Y., Dillen F., Classification of marginally trapped Lagrangian surfaces in Lorentzian complex space forms, J. Math. Phys., 2007, 48(1), 013509, 23 pp.; Erratum, J. Math. Phys., 2008, 49(5), 059901, $1 \mathrm{p}$.

[9] Chen B.-Y., Garay O., Classification of quasi-minimal surfaces with parallel mean curvature vector in pseudo-Euclidean 4-space $\mathbb{E}_{2}^{4}$, Result. Math., 2009, 55(1-2), 23-38.

[10] Chen, B.-Y., Mihai, I., Classification of quasi-minimal slant surfaces in Lorentzian complex space forms, Acta Math. Hungar., 2009, 122(4), 307-328.

[11] Chen B.-Y., Yang D., Addendum to "Classification of marginally trapped Lorentzian flat surfaces in $\mathbb{E}_{2}^{4}$ and its application to biharmonic surfaces", J. Math. Anal. Appl., 2010, 361(1), 280-282.

[12] Ganchev G., Milousheva V., Invariants and Bonnet-type Theorem for Surfaces in $\mathbb{R}^{4}$, Cent. Eur. J. Math. 8 (2010) 993-1008.

[13] Ganchev G., Milousheva V., An Invariant Theory of Marginally Trapped Surfaces in the Fourdimensional Minkowski Space, J. Math. Phys. 53 (2012) Article ID: 033705, 15 pp.

[14] Ganchev G., Milousheva V., Special Classes of Meridian Surfaces in the Four-dimensional Euclidean Space, Bull. Korean Math. Soc. 52, no. 6 (2015), 2035-2045.

[15] Ganchev G., Milousheva V., Meridian Surfaces of Elliptic or Hyperbolic Type in the Four-dimensional Minkowski Space, Math. Commun., 21, no. 1 (2016), 1-21.

[16] Liu H., Liu G., Hyperbolic rotation surfaces of constant mean curvature in 3-de Sitter space, Bull. Belg. Math. Soc. Simon Stevin, 7, no. 3 (2000), 455-466. 
[17] López R., Timelike surfaces with constant mean curvature in Lorentz three-space. Tohoku Math. J. 52 (2000), 515-532.

[18] O'Neill M., Semi-Riemannian geometry with applications to relativity, Academic Press, London 1983.

[19] Rosca, R., On null hypersurfaces of a Lorentzian manifold. Tensor (N.S.) 23 (1972), 66-74.

[20] Sasahara N., Spacelike helicoidal surfaces with constant mean curvature in Minkowski 3-space. Tokyo J. Math. 23 (2000), no. 2, 477-502.

Uluda Ğ University, Department of Mathematics, 16059 Bursa, Turkey

E-mail address: bbulca@uludag.edu.tr

Institute of Mathematics and Informatics, Bulgarian Academy of Sciences, Acad. G. Bonchev Str. Bl. 8, 1113, Sofia, Bulgaria; "L. Karavelov" Civil Engineering Higher School, 175 Suhodolska Str., 1373 Sofia, Bulgaria

E-mail address: vmil@math.bas.bg 